Methods A retrospective cohort analysis of patients was undertaken using prospectively maintained records. Patients commenced on tofacitinib through the patient access scheme between October 2018 to February 2019 were included. Clinical disease activity was measured at baseline, at four and eight weeks using the Simple Clinical Colitis Activity Index (SCCAI). Faecal calprotectin and C-reactive protein were measured at baseline and eight weeks.

Results At the time of submission, 16 patients had commenced tofacitinib, with outcome data available for 8 patients who had reached at least four weeks of treatment. All 8 patients (median age 46) with Mayo 2-3 colitis demonstrated on preinduction endoscopy, were previously exposed to an anti-TNF agent, of which 6 had also failed vedolizumab. Median baseline SCCAI $(n=8)$ fell from 8 (range 2-14) to $3(1-5)$ after four weeks and remained stable at eight weeks. Median baseline faecal calprotectin $(n=5)$ fell from $364(131-645)$ to 95 (30-289). One patient reaching week 16 was in endoscopic remission. Tofacitinib was well tolerated with only one patient reporting a mild headache and diarrhoea, which self-resolved in under a week. No haematological or biochemical abnormalities were noted.

Conclusions Our early experience with tofacitinib for moderate to severe ulcerative colitis is encouraging, with an improvement in SCCAI and faecal calprotectin in all our patients. Oral dosing and a quicker onset of action are other advantages, which may enable positioning above vedolizumab. Further real life data is necessary in this setting to demonstrate effectiveness and a longer term safety profile.

\section{PTH-093 EFFICACY OF THIOPURINE MONOTHERAPY IN THE UK INFLAMMATORY BOWEL DISEASE BIORESOURCE COHORT}

${ }^{1}$ You Yi Hong* ${ }^{*}$ 1,2Laetitia Pele, ${ }^{1,2}$ Rachel Simpkins, ${ }^{1,2}$ Catherine Thorbinson,
${ }^{1,2}$ Deepthy Francis, ${ }^{2}$ Rasha Shawky, 1,2Miles Parkes. 'Department of Medicine, University of
Cambridge, Cambridge, UK; ${ }^{2}$ IBD Bioresource, NIHR BioResource, Cambridge, UK

10.1136/gutjnl-2019-BSGAbstracts.152

Introduction IBD BioResource is currently recruiting patients with Crohn's Disease (CD), Ulcerative Colitis (UC) and IBDU from 89 hospitals UK-wide. To date $>19,000$ patients have been recruited with detailed clinical phenotype data plus serum and DNA, and all will have had Genome-Wide Association Scans \pm whole genome sequencing by April 2019. Subjects can be recalled by genotype or phenotype for downstream studies by any investigator.

Thiopurines play a key role in the management of IBD used either as monotherapy or in combination with other treatments to maintain remission. However, data regarding long term effectiveness are sparse.

Aims To characterise the long term effectiveness of thiopurine monotherapy among subjects in the IBD BioResource cohort

Method IBD phenotype data were extracted by research nurses and clinicians in each hospital site following case note review and uploaded to a Redcap database. Response to treatment was empirically classified as 'effective', 'not effective', and 5 other categories ('transient', 'partial', 'intolerant' etc.). By interrogating the Redcap database and analysing the data in $\mathrm{R}$ we sought to identify the proportion of patients in whom thiopurine was effective as monotherapy - in whom
(1) treatment was classified as 'effective' and (2) there had been no escalation to biologic therapy or need for surgery for the duration of thiopurine therapy. Patients started on anti TNF therapy at thiopurine initiation or undergoing surgery in the 1 year prior to initiation were excluded since we could not assess effectiveness of thiopurine monotherapy in these groups.

Results Data were available on 8296 IBD BioResource subjects $(48.3 \%$ male) treated with a thiopurine and meeting inclusion criteria for assessment. In 2417 patients (29.1\%) thiopurine monotherapy had been deemed to be an effective maintenance treatment - meeting both criteria (1) and (2) above. Long term effectiveness was higher in UC/IBDU $(1531 / 3485 ; 43.9 \%)$ compared to CD $(883 / 4799 ; 18.4 \%$ Chi Sq $\mathrm{P}<0.0001)$. 3459/8296 (41.7\%) treated with thiopurine were started within 1 year of diagnosis (Figure 1). Mean duration on drug $=5.9$ years at the time of IBD BioResource recruitment (at least $78 \%$ of those in whom thiopurine monotherapy has been effective are still on this therapy).

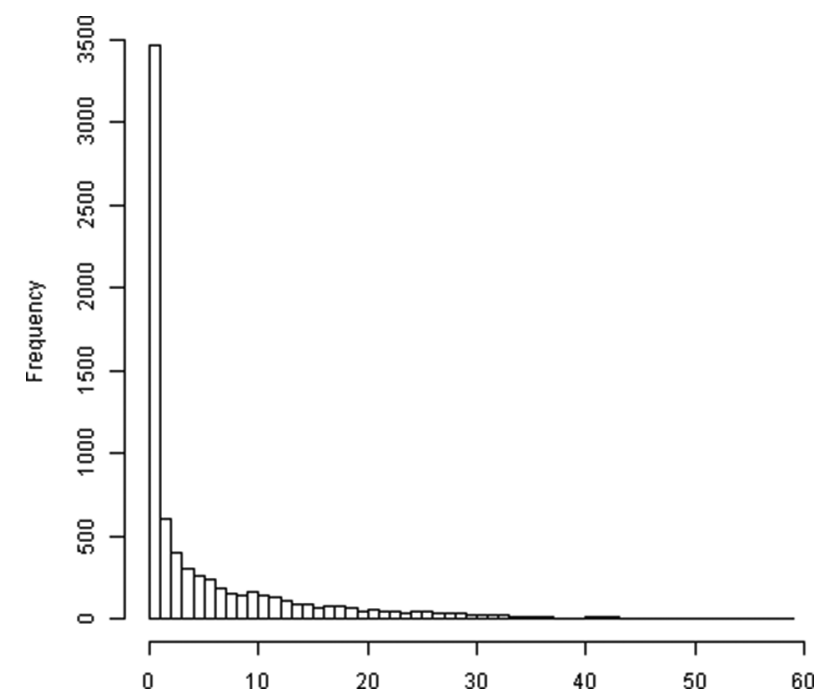

Abstract PTH-093 Figure 1 Time (years) from diagnosis to thiopurine initiation

Conclusion Thiopurines can be effective in producing durable remission, particularly in UC. Pharmacogenetic studies will follow. The IBD BioResource is open to all investigators for recall of well characterised patient cohorts.

\section{PTH-094 THIOPURINE ADVERSE EVENTS IN PATIENTS WITH INFLAMMATORY BOWEL DISEASE IN THE UK- IBD BIORESOURCE COHORT}

You Yi Hong*, Achini Diana Withanachchi, Rasha Shawky, Miles Parkes. Addenbrooke's Hospital, Cambridge, UK

\subsection{6/gutjnl-2019-BSGAbstracts.153}

Introduction The Inflammatory Bowel Disease (IBD) BioResource is recruiting patients with Crohn's Disease (CD), Ulcerative Colitis (UC) or IBD type Unclassified (IBDU) from 89 hospitals UK-wide. $>19,000$ subjects have been recruited to 